\title{
Optical Reflectance Measurements for Commonly Used Reflectors
}

\author{
Martin Janecek, Member, IEEE, and William W. Moses, Fellow, IEEE
}

\begin{abstract}
When simulating light collection in scintillators, modeling the angular distribution of optical light reflectance from surfaces is very important. Since light reflectance is poorly understood, either purely specular or purely diffuse reflectance is generally assumed. In this paper we measure the optical reflectance distribution for eleven commonly used reflectors. A $440 \mathrm{~nm}$, output power stabilized, un-polarized laser is shone onto a reflector at a fixed angle of incidence. The reflected light's angular distribution is measured by an array of silicon photodiodes. The photodiodes are movable to cover $2 \pi$ of solid angle. The light-induced current is, through a multiplexer, read out with a digital multimeter. A LabVIEW program controls the motion of the laser and the photodiode array, the multiplexer, and the data collection. The laser can be positioned at any angle with a position accuracy of 10 arc minutes. Each photodiode subtends $6.3^{\circ}$, and the photodiode array can be positioned at any angle with up to 10 arc minute angular resolution. The dynamic range for the current measurements is $10^{5}: 1$. The measured light reflectance distribution was measured to be specular for several ESR films as well as for aluminum foil, mostly diffuse for polytetrafluoroethylene (PTFE) tape and titanium dioxide paint, and neither specular nor diffuse for Lumirror ${ }^{\circledR}$, Melinex ${ }^{\circledR}$ and Tyvek®. Instead, a more complicated light distribution was measured for these three materials.
\end{abstract}

Index Terms - Lambertian reflection, light collection, Monte Carlo methods

\section{INTRODUCTION}

$\mathrm{T}^{\circ}$ accurately predict light distribution in Monte Carlo simulations, e.g. by using DETECT [1], [2], Litrani [3], Geant4 [4], [5], or GATE [6-9] Monte Carlo software, surface reflections have to be well understood. Studies of surface reflection can be approached by 1) physical optics models [1016] (which use electromagnetic wave theory to analyze the reflection of incident light), 2) geometrical optics models [1720] (which use the short wavelength - compared to the surface irregularities - to simplify the physical model), or 3) measuring the actual angular distribution. The theoretical

Manuscript received January 15, 2008. This work was supported by the National Nuclear Security Administration, Office of Defense Nuclear Nonproliferation, Office of Nuclear Nonproliferation Research and Engineering (NA-22) of the U.S. Department of Energy under Contract No. DE-AC02-05CH1 1231, grant number NNSA LB06-316-PD05 / NN2001000.

M. Janecek is with the Lawrence Berkeley National Laboratory, Berkeley, CA 94720 USA (phone: 510-486-5579; fax: 510-486-4768; e-mail: mjanecek@lbl.gov).

W. W. Moses is with the Lawrence Berkeley National Laboratory, Berkeley, CA 94720 USA (e-mail: wwmoses@lbl.gov). models classify reflections into linear combinations of specular reflection and diffuse reflection. For a review of the various theoretical reflection models, we refer the reader to a detailed description by Nayer et al. [21]. Although the theoretical models are fairly successful at predicting the degree of diffuse reflection versus specular reflection, the results are estimates of the real world. Furthermore, surface characteristics are not always known and many materials used as reflectors are designed to enhance special optical features that have not been incorporated into the models in the Monte Carlo software. Existing Monte Carlo simulation code therefore allows the surface properties to be set to either specular, diffuse, or a linear combination of the two, and more advanced settings are not available.

The aim of this work is to investigate the angular distribution produced for light reflected by commonly used reflectors. The results will be incorporated into existing Monte Carlo models and aid in improving Monte Carlo simulations for air-coupled reflectors.

\section{BACKGROUND}

\section{A. Specular versus diffuse reflection}

Specular (mirror-like) reflection occurs when a surface is very smooth. An incident ray of light is reflected by the surface into a single ray of light with the reflection angle being equal to the incident angle. Diffuse reflection, on the other hand, is produced from rough surfaces and from scattering from inside of the reflector material and is characterized by the light being reflected through a broad distribution of angles, even for a single angle of incidence. A special case of diffuse reflection is a Lambertian surface, which by definition appears equally bright from all viewing angles. Another way of stating this is that the emitted light from the reflection surface is proportional to the cosine of the angle between the surface normal and the angle of observation. This is known as Lambert's cosine law. Since the surface area seen from the same angle of observation is the product of the surface area and the cosine of the same angle, and light intensity is equal to emitted light divided by area, the two cosines cancel out, which leads to the original definition of a Lambertian surface.

What this means for our measurements is that although a Lambertian surface will appear equally bright from all viewing angles, for Lambertian surfaces we should measure a cosine function, as illustrated in Fig. 1, independent of the 
incident angle.

\section{METHODS}

\section{A. Instrument}

An instrument for measuring the angular distribution of reflectance has been designed and built [22]. Table I displays some important characteristics of the instrument and a mechanical drawing is displayed in Fig 2.

A laser beam, which can be rotated from theta $-90^{\circ}$ to $+90^{\circ}$, is aimed towards the center of a $50.8-\mathrm{mm}$ diameter plate, which holds a reflector sample. The laser beam is reflected by the reflector and the reflected light distribution is measured by a movable semi-circular array of photodiodes. The array can (independently) be rotated from theta $-90^{\circ}$ to $+90^{\circ}$ and can thereby measure the angular distribution over the entire $2 \pi$ solid angle.

The laser is a 440-nm un-polarized $\mathrm{TEM}_{00}$ laser with $<0.8 \mathrm{~mm}$ beam diameter and $1.0 \%$ power stability over 48 hours. The array consists of 36 Hamamatsu [Japan] S8729 PIN photodiodes. The $4 \times 5 \mathrm{~mm}^{2}$ photodiodes are mounted in two rows, offset to each other by half the length of a photodiode, with each diode subtending $6.3^{\circ}$. All photodiodes are mounted at the same distance from the reflection spot and cover equal solid angles. The currents from the photodiodes are switched through a multiplexer to a digital multimeter, where the current is recorded. The current measurement has a dynamic range of $10^{5}: 1$. A LabVIEW program controls the motion of the laser and photodiodes, the multiplexer switch, and the data collection. The mechanical set-up is placed inside of a light-tight box.

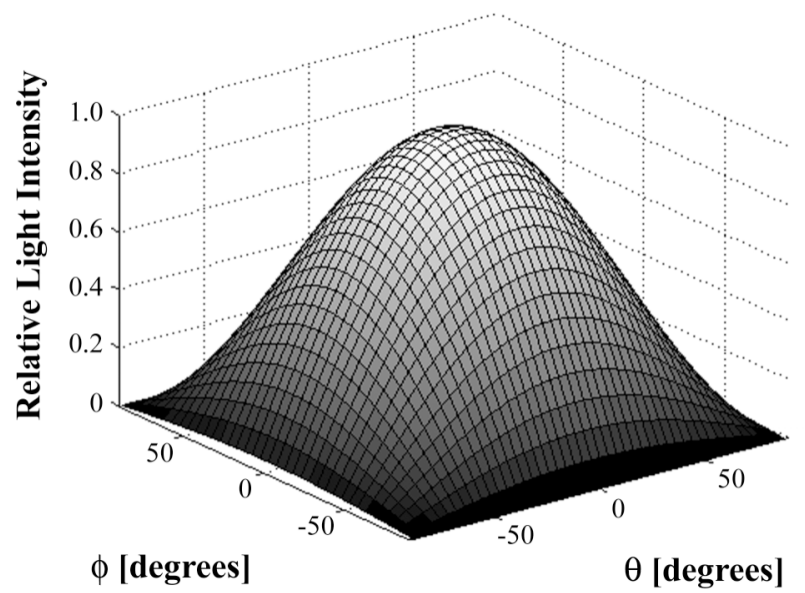

Fig. 1. Theoretical Lambertian angular distribution. The coordinate system is defined in Fig. 2.

TABLE I

INSTRUMENT CHARACTERISTICS

\begin{tabular}{lc}
\hline \hline Laser wavelength & $440 \mathrm{~nm}$ \\
\hline Laser power stability & $< \pm 1 \%$ over 48 hours \\
\hline Sampling & $4^{\circ}$ (theta) $\times 5^{\circ}($ phi) \\
\hline Angular resolution & $<1^{\circ}$ \\
\hline Dynamic range & $10^{5}: 1$ \\
\hline
\end{tabular}

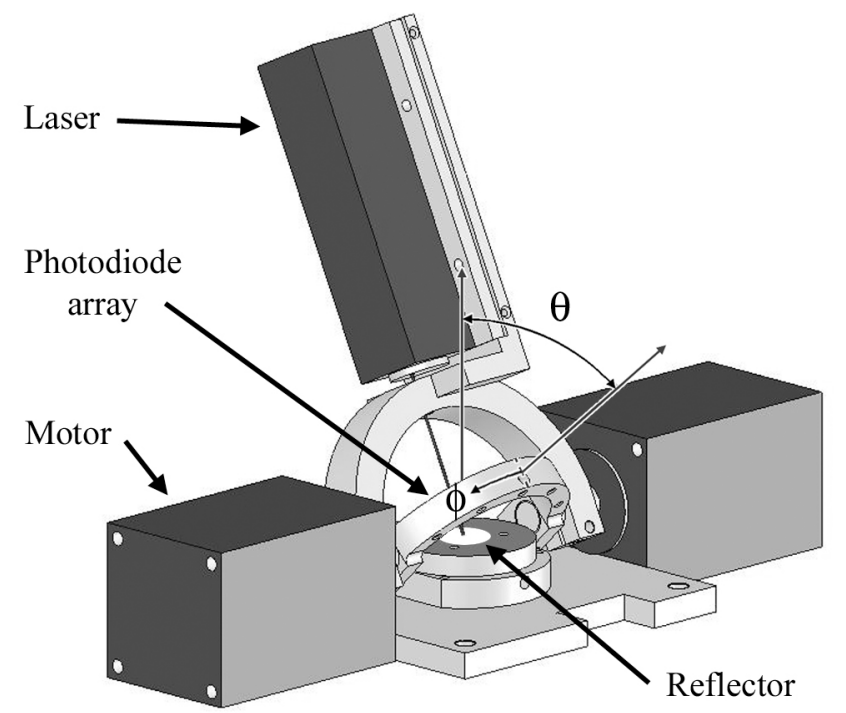

Fig. 2. Scale drawing of the instrument for measuring the light distribution. A laser, mounted on an arch, is aimed towards a center spot. The laser can, with a stepper motor, be rotated from theta $=-90^{\circ}$ to $+90^{\circ}$. The photodiode array is mounted on a second arch, and can be rotated from theta $=-90^{\circ}$ to $+90^{\circ}$ with a second stepper motor. The theta and phi angles display the coordinates for the detectors, which are mounted on the detector arch. The entire set-up can fit inside of a $380 \times 370 \times 220 \mathrm{~mm}^{3}$ box (width $\times$ depth $\times$ height), with full rotational freedom for the laser.

\section{B. Methods}

Each reflector sample was examined for laser incidence angles from $+2^{\circ}$ to $+82^{\circ}$ in steps of $4^{\circ}$. For each laser incidence angle, the photodiode array was stepped from theta $-90^{\circ}$ to $+90^{\circ}$ with $4^{\circ}$ steps. At each photodiode theta angle, all 36 photodiodes were read out. Each photodiode measurement was averaged for $3 \mathrm{~s}$. (The digital multimeter reads the current at discrete times, so $3 \mathrm{~s}$ acquisition time is equivalent to 10 single consecutive measurements). The data was saved for each laser angle in separate text-files for post-processing.

To eliminate the effect of the dark current and any stray light, a background subtraction was performed for all the acquired data. The background was measured in a separate acquisition with a black hole in the center of the set-up. The black hole was simply a hole through a black cloth with more black cloth covering the insides of the hole.

We use a $4^{\circ}$ (theta) $\times 5^{\circ}$ (phi) angular grid to display our results. The photodiode centers are located $5^{\circ}$ apart, while a photodiode covers $4^{\circ}$ in the theta direction. Because of this, we chose the theta step size for the photodiode arch to be $4^{\circ}$, as previously mentioned at the beginning of this section. Our results, after each photodiode precise location was calculated for each current measurement, were rebinned to this $4^{\circ} \times 5^{\circ}$ grid.

Linear interpolation was thereafter performed for any unsampled areas. Un-sampled areas occur when the laser and the detector arch are at the same value of theta, as the detector arch blocks the incident laser beam. When this occurs, signals that vary rapidly with angle (e.g., specular reflection) cannot be accurately determined. However, slower varying functions (e.g., diffuse distributions) can still be estimated with linear interpolation. We have removed some material from the support arch for the photodiode array to minimize the range of 
the un-sampled angles.

Finally, the reflectivity coefficient was calculated for each laser angle by integrating the measured light over the $2 \pi$ of solid angle. The reflectivity was normalized to the value of the four layer thick Teflon $^{\mathbb{B}}$ tape. For those reflectors that exhibited a combination of Lambertian and specular reflection (titanium dioxide paint and Teflon ${ }^{\circledR}$ tape), the proportions between the two were calculated by integrating the signal in each light component. This was done by fitting a cosinefunction to the angular distribution with the least square method and using the fitted function to calculate the Lambertian portion of the signal. The remaining part of the signal was assumed to be the specular portion of the signal.

\section{Reflector materials}

We examined a total of eleven samples of reflective materials. All the reflector samples were placed on a glass slide to ensure a flat and horizontal surface.

We measured four specular reflectors; aluminum foil [Kaiser Foil, Northbrook, IL] measuring $30 \mu \mathrm{m}$ in thickness, and three separate versions of ESR (Enhanced Specular Reflector) film by 3M [St. Paul, MN]: VM2000, VM2002, and VM3000. The VM-series is the old labeling system for the ESR films and the number indicates its generation. Each film had its protective coating removed before measurements were performed, and was $65 \mu \mathrm{m}$ in thickness.

We performed measurements on three diffuse reflectors. White titanium dioxide paint [NE560, Nuclear Enterprises, Inc., San Carlos, CA] was evenly painted onto a glass slide and allowed to dry before measurements. The paint thickness was 75-100 $\mu \mathrm{m}$. We examined two types of polytetrafluoroethylene (PTFE) tapes, a well-known Lambertian material [23-26]. The two examined versions were a matte Teflon ${ }^{\circledR}$ tape purchased at ACE Hardware [Oak Brook, IL] and a $90 \mu \mathrm{m}$ thick glossy PTFE tape (unknown origin). We measured three layers $(230 \mu \mathrm{m})$ and four layers $(310 \mu \mathrm{m})$ of the ACE Teflon ${ }^{\circledR}$ tape, while the glossy tape was measured as a single layer.

Tyvek $^{\circledR}$ paper $\left[\right.$ DuPont $^{\mathrm{TM}}$, Wilmington, DE] measuring $150 \mu \mathrm{m}$ in thickness, Lumirror ${ }^{\circledR}$ [Toray, Japan] measuring $240 \mu \mathrm{m}$ in thickness, and Melinex ${ }^{\circledR}$ [DuPont $\left.{ }^{\mathrm{TM}}\right]$ measuring $125 \mu \mathrm{m}$ in thickness were also characterized.

\section{RESUlTS}

The angular distributions for incidence angle theta equal to $50^{\circ}$ are displayed in Fig. 3 for some selected samples. The results are displayed as contour plots with 8 isometric lines. Figs. 4-11 display center profiles $\left(\mathrm{phi}=0^{\circ}\right.$ ) of the angular distribution for the various reflectors for selected incidence angles.

All the ERS films, both back and front sides, showed comparable specular reflection. The angular distribution for VM2000 film is shown in Fig. 3A. For incident angle $14^{\circ}$ and greater, the specular reflection peak has a constant intensity at the reflection angle equal to the incident angle, as shown in Fig. 4. Below this angle, the peak falls in the un-sampled areas, and cannot be measured with our setup.

Aluminum foil exhibited specular reflection similar to the ESR films, but with a wider angular width of the specular peak, as can be seen in Fig. 3B. The integrated light intensity in the specular peak is constant and as we increased the incidence angle, the width of the reflection peak decreased, increasing the height of the peak, see Fig. 5.

Titanium dioxide displayed a Lambertian distribution for incidence angles up to $50^{\circ}$, as shown in Figs. $3 \mathrm{C}$, and 6. Above $50^{\circ}$ a specular peak started developing and the specular peak increased in strength with increasing incident angles while the diffuse portion decreased. The specular portion of the light distribution is less than $1 \%$ below incidence angles of $50^{\circ}$, but grows as high as $30 \%$ at large incidence angles, as can be seen in Fig. 12.

The PTFE tapes exhibited a nearly pure Lambertian distribution, see Figs. 3D, 7, and 8. For the three-layer thick, as well as for the four-layer thick, ACE Teflon ${ }^{\circledR}$ tape there is an additional small specular peak that is relatively broad. The light intensity in this specular peak is roughly $2 \%$ for incidence angles below $40^{\circ}$. Above $40^{\circ}$, the specular peak grows, again becoming as large as $25 \%-30 \%$ for large incidence angles, as can be seen in Figs. 7 and 12. The same observations are true for the $90 \mu \mathrm{m}$ thick glossier PTFE tape, with the exception of the specular peak being narrower and much larger in amplitude. However, the total light intensity in the specular peak is about $2 \%$ for low incidence angles, approximately the same as for the ACE Teflon ${ }^{\circledR}$ tape, as can be seen in Fig. 12. Figs. 3D and 7 display the angular light distribution for three layer thick ACE Teflon ${ }^{\circledR}$ tape, while Fig. 8 displays the distribution for glossy PTFE tape.

The Tyvek ${ }^{\circledR}$ paper displayed a distribution that is neither specular, diffuse, nor a linear combination of the two distributions, as can be seen in Figs. $3 \mathrm{E}$ and 9. The geometrical shape of the distribution is roughly triangular, with its peak located at the reflection angle roughly equal to the incidence angle.

Lumirror $^{\mathbb{B}}$ and Melinex ${ }^{\mathbb{R}}$ have very similar reflection distribution shapes. As with Tyvek ${ }^{\circledR}$, neither of them displays a linear combination of specular and diffuse reflection distributions. The distribution can instead be described as a sharp volcano shape, or a needle poking up through a very flexible membrane. The reflection peaks for Lumirror $^{\circledR}$ are about twice as wide compared to Melinex ${ }^{\circledR}$, s peaks. The distribution for Lumirror ${ }^{\circledR}$ is displayed in Figs. $3 \mathrm{~F}$ and 10 , while the distribution for Melinex ${ }^{\circledR}$ is displayed in Fig. 11.

For all reflector samples, the reflectivity coefficient was calculated for all incidence angles. It was measured to be constant for all incident angles between $14^{\circ}$ and $78^{\circ}$. Based on this, we calculated each reflector's reflection coefficient as the average for all of these angles. The reflection coefficient was normalized to the value of the four layer thick Teflon ${ }^{\circledR}$ tape, as this recorded the highest light reflectance. The results are displayed in Table II. 

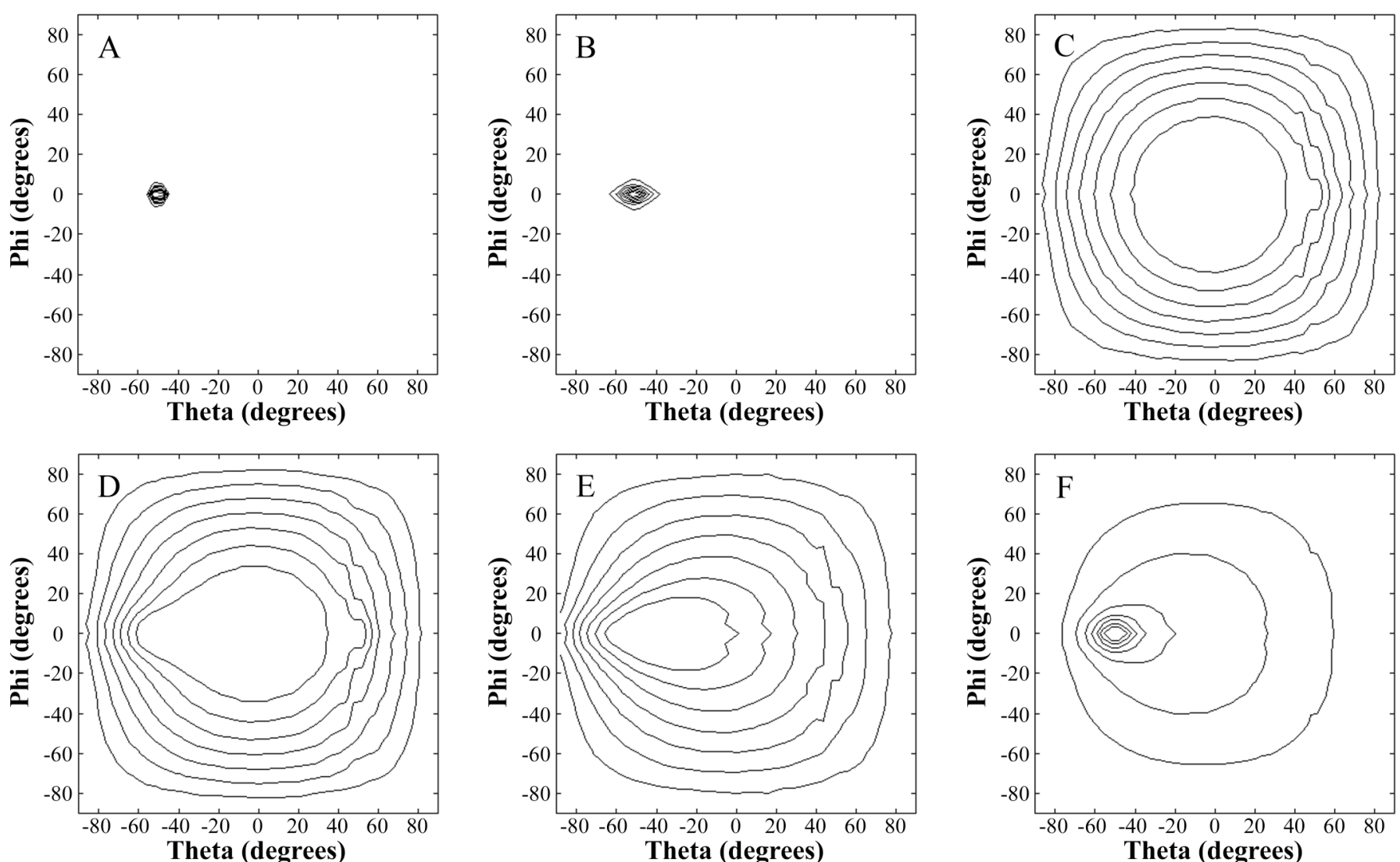

Fig. 3. Angular distributions for selected reflectors. All shown data are for laser incidence angle of theta equal to $50^{\circ}$. Each data set is shown with a contour plot with 8 equally distant isometric lines. A) VM2000 film (All the ESR films showed same distribution, both front side and back side, and VM2000 is shown to illustrate the distribution), B) aluminum foil, C) titanium dioxide paint, D) three layers of Teflon ${ }^{\circledR}$ tape, E) Tyvek ${ }^{\circledR}$ paper, and F) Lumirror ${ }^{\circledR}$.

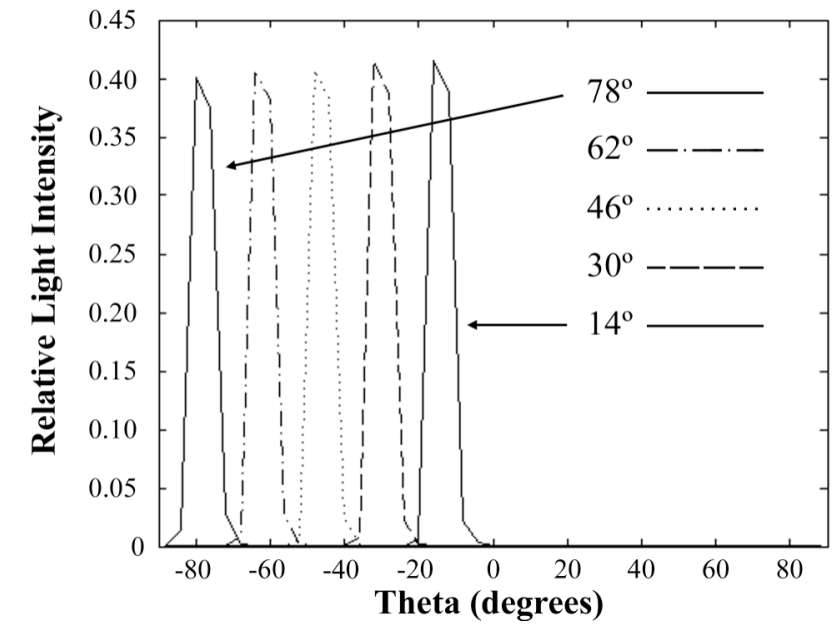

Fig. 4. VM2000 film's angular distribution profiles for phi equal to $0^{\circ}$ and for incidence angles of $14^{\circ}$ to $78^{\circ}$ (in $16^{\circ}$ steps).

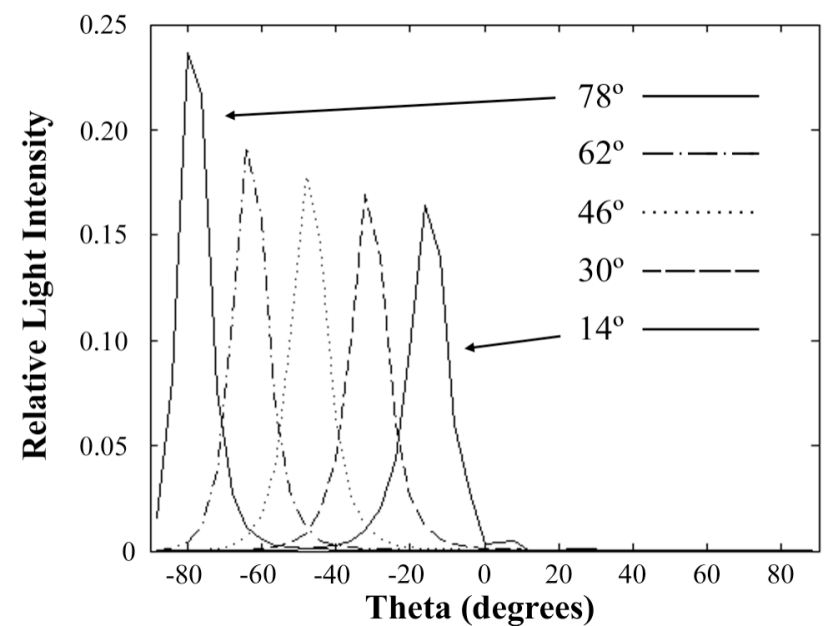

Fig. 5. Aluminum foil's angular distribution profiles for phi equal to $0^{\circ}$ and for incidence angles of $14^{\circ}$ to $78^{\circ}$ (in $16^{\circ}$ steps). 


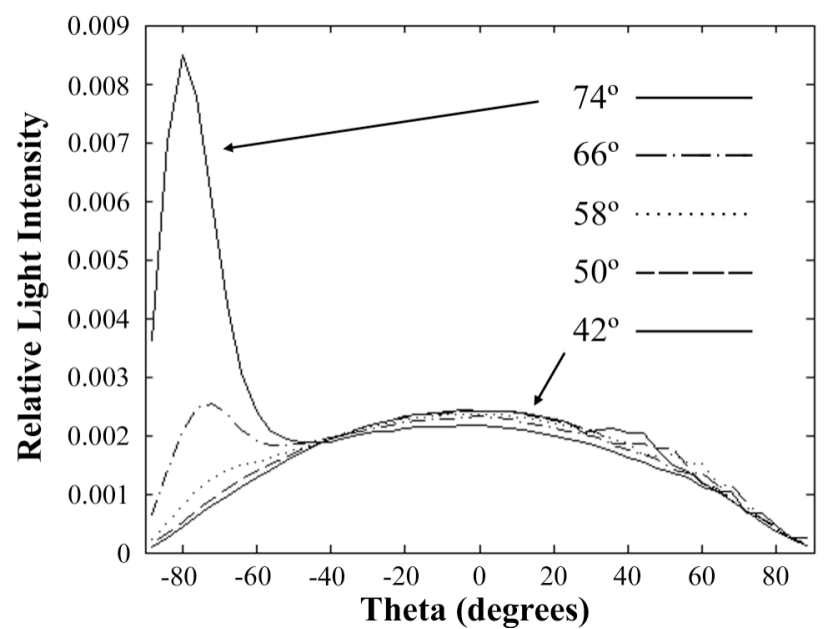

Fig. 6. Titanium dioxide's angular distribution profiles for phi equal to $0^{\circ}$ and for incidence angles of $42^{\circ}$ to $74^{\circ}$ (in $8^{\circ}$ steps).

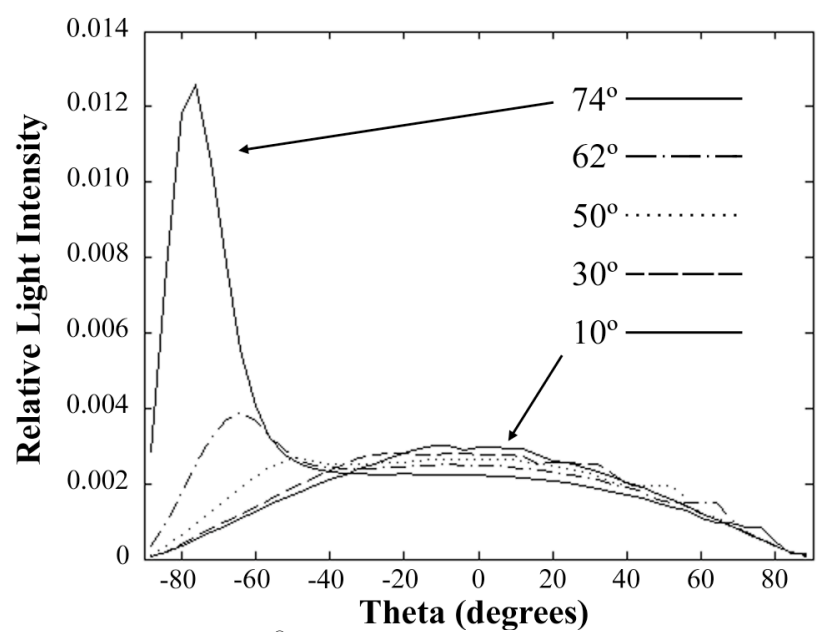

Fig. 7. Three layer Teflon ${ }^{\circledR}$ tape's angular distribution profiles for phi equal to $0^{\circ}$ and for incidence angles of $10^{\circ}, 30^{\circ}, 50^{\circ}, 62^{\circ}$ and $74^{\circ}$.

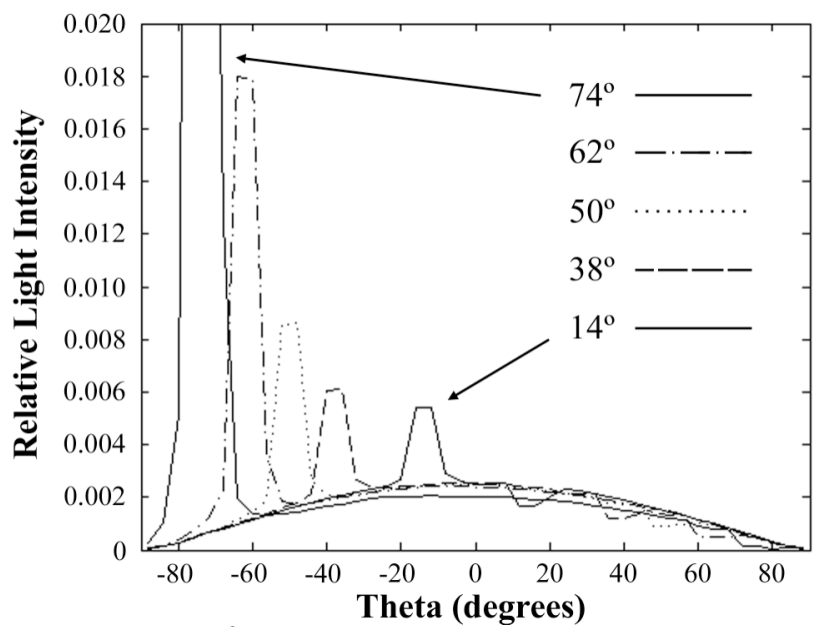

Fig. 8. Glossy Teflon ${ }^{\circledR}$ tape's angular distribution profiles for phi equal to $0^{\circ}$ and for incidence angles of $14^{\circ}, 38^{\circ}, 50^{\circ}, 62^{\circ}$ and $74^{\circ}$. The peak for incidence angle $74^{\circ}$ reaches 0.057 on the "Relative Light Intensity" scale.

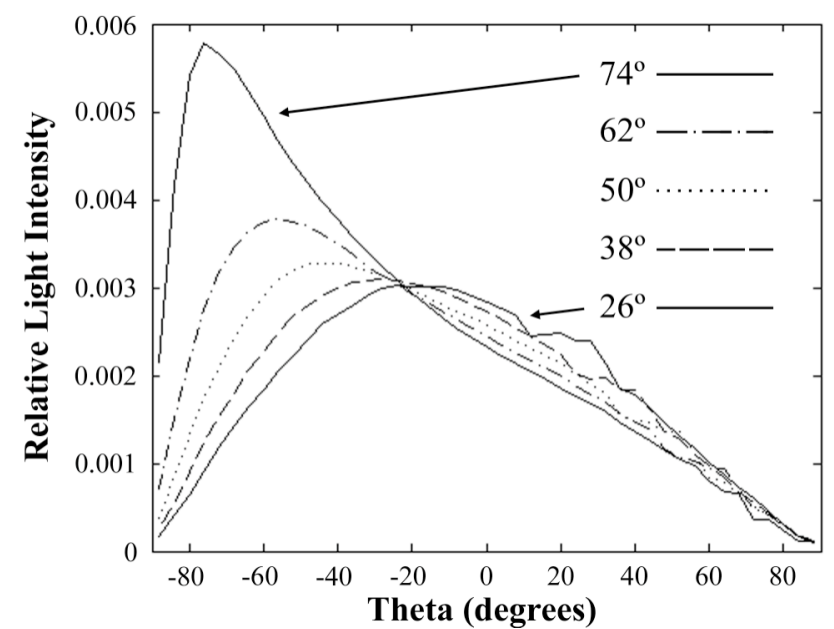

Fig. 9. Tyvek ${ }^{\circledR}$ paper's angular distribution profiles for phi equal to $0^{\circ}$ and for incidence angles of $26^{\circ}$ to $74^{\circ}$ (in $12^{\circ}$ steps).

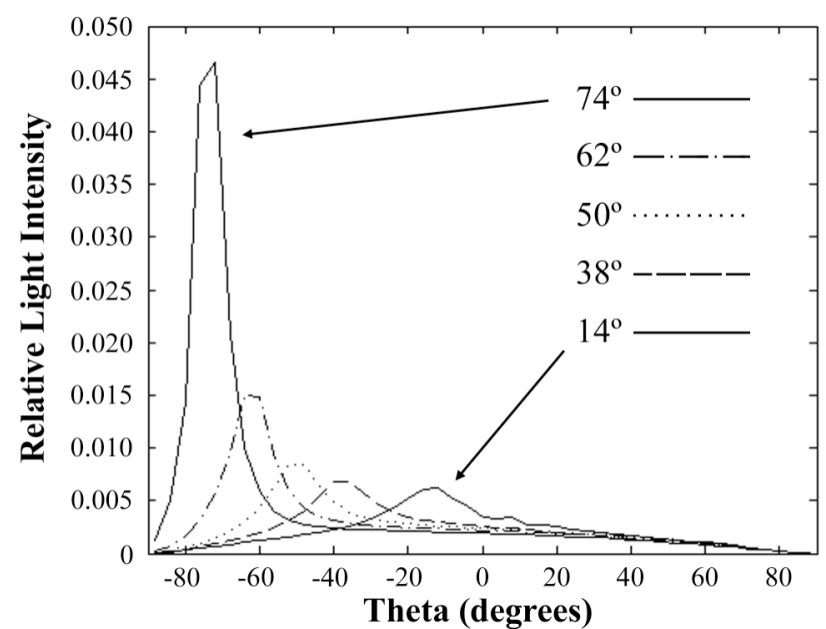

Fig. 10. Lumirror ${ }^{\circledR}$ s angular distribution profiles for phi equal to $0^{\circ}$ and for incidence angles of $14^{\circ}, 38^{\circ}, 50^{\circ}, 62^{\circ}$ and $74^{\circ}$.

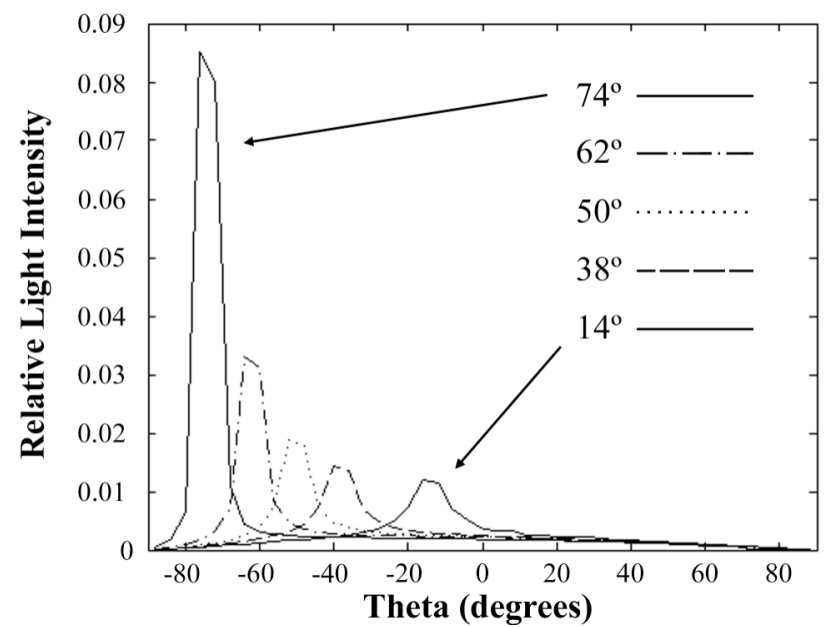

Fig. 11. Melinex ${ }^{\circledR}$ s angular distribution profiles for phi equal to $0^{\circ}$ and for incidence angles of $14^{\circ}, 38^{\circ}, 50^{\circ}, 62^{\circ}$ and $74^{\circ}$. 


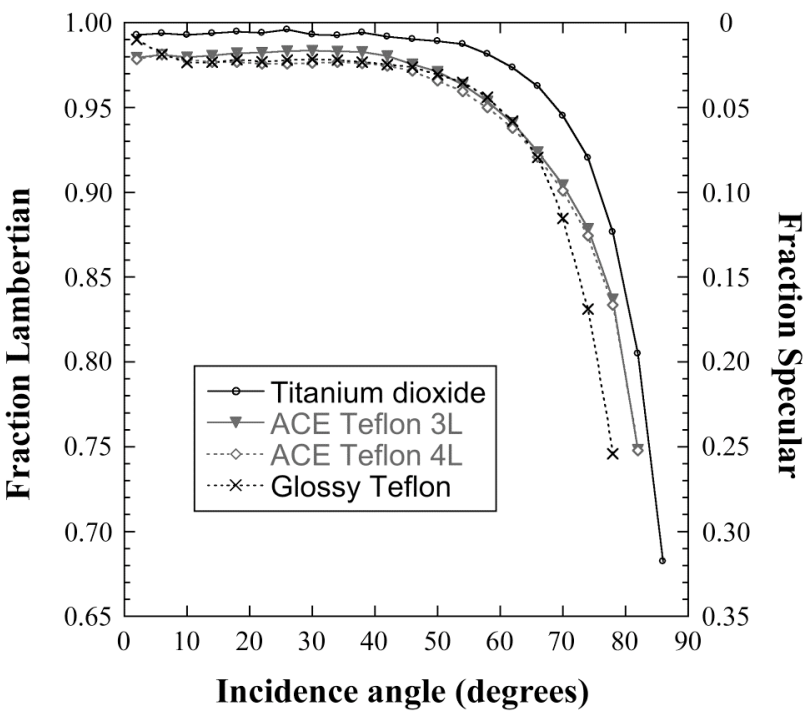

Fig. 12. Fraction of Lambertian (left y-axis) and specular (right y-axis) reflection as a function of incidence angle for the reflectors that exhibited a combination of Lambertian and specular reflections. Notice that the y-axis on the left and the $y$-axis on the right add up to $100 \%$.

TABLE II

RELATIVE REFLECTION COEFFICIENTS

\begin{tabular}{|c|c|}
\hline Reflector & Refl. coeff. \pm St. dev. \\
\hline Teflon $^{(R}$ - 3 layers & $0.992 \pm 0.004$ \\
\hline Teflon $^{\circledR}$ - 4 layers & $1.000 \pm 0.006$ \\
\hline Glossy PTFE & $0.934 \pm 0.004$ \\
\hline VM2000 & $0.944 \pm 0.013$ \\
\hline VM2002 & $0.960 \pm 0.007$ \\
\hline VM3000 & $0.890 \pm 0.019$ \\
\hline Aluminum foil & $0.787 \pm 0.014$ \\
\hline Tyvek $^{(\mathbb{R})}$ paper & $0.979 \pm 0.004$ \\
\hline Lumirror $^{\left({ }^{2}\right.}$ & $0.990 \pm 0.006$ \\
\hline Melinex $^{\circledR}$ & $0.969 \pm 0.003$ \\
\hline Titanium dioxide paint & $0.951 \pm 0.003$ \\
\hline
\end{tabular}

\section{CONClusions}

We have measured the angular distribution of the reflection from commonly used reflector materials. All distributions are smoothly varying functions. ESR films and aluminum foil were measured to be specular reflectors. Titanium dioxide paint and Teflon ${ }^{\circledR}$ tape were measured to be Lambertian reflectors, although a specular component appeared at large incident angles. Theoretical models predict these specular peaks, for instance the Beckmann-Spizzichino model [10]. Lumirror $^{\circledR}$, Melinex ${ }^{\circledR}$, and Tyvek ${ }^{\circledR}$ paper cannot be described as specular reflectors, diffuse reflectors, nor linear combination of the two, but instead more complex reflection distributions were measured. These results suggest that existing Monte Carlo methods are unlikely to accurately simulate anything other than specular reflections, and may explain the difficulties in validating these models.

The reflectivity coefficients were calculated from the measured data, and most reflectors had reflection coefficients within a few percent of each other. PTFE exhibited the highest reflection coefficient, which has been measured by others [2325 ] to be $99 \%$ at $440 \mathrm{~nm}$.

It is not possible for the casual user to include the measured data into existing Monte Carlo simulations, as non-trivial Monte Carlo code modifications are necessary. We are planning to make these modifications and hope to make them part of the standard Monte Carlo packages. This will aid in creating more accurate Monte Carlo simulation models.

\section{ACKNOWLEDGMENT}

This work was supported by the National Nuclear Security Administration, Office of Defense Nuclear Nonproliferation, Office of Nuclear Nonproliferation Research and Engineering (NA-22) of the U.S. Department of Energy under Contract No. DE-AC02-05CH11231, grant number NNSA LB06-316-PD05 / NN2001000.

\section{REFERENCES}

[1] G.F. Knoll, T.F. Knoll, and T.M. Henderson, "Light collection in scintillating detector composites for neutron detection," IEEE Trans. Nucl. Sci., pt. 1, vol. 35, pp. 872-875, Feb. 1988.

[2] F. Cayouette, D. Laurendeau, and C. Moisan, "DETECT2000: An Improved Monte-Carlo Simulator for the Computer Aided Design of Photon Sensing Devices", Proc. SPIE Photonic North, Quebec, Canada, June 2002

[3] F.-X. Gentit, "Litrani: a general purpose Monte-Carlo program simulating light propagation in isotropic or anisotropic media", Nucl. Instr. and Meth. A, vol. 486, no. 1-2, pp., p. 35, June 2002

[4] J. Allison, et al., "Geant4 Developments and Applications", IEEE Trans. Nucl. Sci., vol. 53, no. 1, pp. 270-278, February 2006

[5] S. Agostinelli, et al., "Geant4 - A Simulation Toolkit", Nucl. Instr. and Meth. A, vol. 506, pp. 250-303, 2003

[6] G. Santin, D. Strul, D. Lazaro, et al., "GATE: A Geant4-Based Simulation Platform for PET and SPECT Integrating Movement and Time Management", IEEE Trans. Nucl. Sci., vol. 50, no. 5, pp. 15161521 , Oct. 2003

[7] D. Strul, G. Santin, D. Lazaro, et al., "GATE (Geant4 Application for Tomographic Emission): a PET/SPECT general-purpose simulation platform”, Nucl. Phys. B (Proc. Suppl.), vol. 125, pp. 75-79, 2003

[8] K. Assie, V. Breton, I. Buvat, et al., "Monte Carlo simulation in PET and SPECT instrumentation using GATE", Nucl. Instr. and Meth. in Phys. Research A, vol. 527, pp. 180-189, 2004

[9] S. Jan, G. Santin, D. Strul, et al., "GATE: a simulation toolkit for PET and SPECT", Phys. Med. Biol., vol. 49, pp. 4543-4561, 2004

[10] P. Beckmann and A. Spizzichino, "The scattering of electro-magnetic waves from rough surfaces", The Macmillan Company, 1963

[11] S.O. Rice, "Reflection of electromagnetic waves from slightly rough surfaces", Commun. Pure Appl. Math., Vol. 4, pp. 361-378, 1951

[12] H. Davies, "The reflection of electromagnetical waves from rough surfaces", Proc. Inst. Electr. Eng., vol. 101, pp. 209-214, 1954

[13] A.K. Fung, Z. Li, and K.S. Chen, "Backscattering from a randomly rough dielectric surface", IEEE Trans. Geosci. Remote Sens., vol. 30, no. 2, pp. 356-369, 1992

[14] A.K. Fung, G.W. Pan, "A scattering model for perfectly conducting random surfaces I. Model development", Int. J. Remote Sens., vol. 8, no. 11, pp. 1579-1593, 1987

[15] L. Tsang, J.A. Kong, K.H. Ding, and C.O. Ao, "Scattering of Electromagnetic Waves, Numerical Simulations", Wiley, 2001

[16] M. Saillard and A. Sentenac, "Rigorous solutions for electromagnetic scattering from rough surfaces ", Waves in Random Media, vol. 11, pp. 103-137, 2001

[17] K. Torrance and E. Sparrow, "Theory for off-specular reflection from roughened surfaces", Journal of the Optical Society of America, no. 57, pp. 1105-1114, 1967

[18] T. Whitted, "An improved illumination model for shaded display", Commun. ACM, vol. 23, no. 6, pp 343-349, June 1980 
[19] B.T. Phong, "Illumination for computer generated pictures", Commun. $A C M$, vol. 18 , no. 6 , pp. 311-317, June 1975

[20] J.F. Blinn, "Models of light reflection for computer synthesized pictures", Computer Gr., vol. 11, no. 2, pp. 192-198, 1977

[21] S.K. Nayer, K. Ikeuchi, and T. Kanade, "Surface reflection: Physical and geometrical considerations", IEEE Transactions on Pattern Analysis and Machine, vol. 13, no. 7, July 1991

[22] M. Janecek and W.W. Moses, "Design of an Instrument to Measure Optical Reflectance of Scintillating Crystal Surfaces", IEEE Trans. Nucl. Sci., (accepted for publication, in press)

[23] Labsphere, Reflections Newsletter, pp. 1-4, Sept. 1998

[24] V.R. Weidner and J.J. Hsia, "Reflection Properties of Pressed Polytetrafluoroethylene Powder", Journal of Optical Society of America, vol. 71, no. 7, pp. 856-861, 1981

[25] B. Waldwick, C. Chase, and B. Chang, "Increased efficiency and performance in laser pump chambers through use of diffuse highly reflective materials", SPIE Proceedings, vol. 6663, 2007

[26] B. Courreges-Lacoste, J.G. Schaarsberg, R. Sprik, and S. Delwart, "Modeling of Spectralon diffusers for radiometric calibration in remote sensing”, Opt. Eng., vol. 42, no. 12, pp. 3600-3607, December 2003 